\title{
Variables that influence Ironman triathlon performance - what changed in the last 35 years?
}

This article was published in the following Dove Press journal:

Open Access Journal of Sports Medicine

25 August 2015

Number of times this article has been viewed

\author{
Beat Knechtle $e^{1,2}$ \\ Raphael Knechtle ${ }^{2}$ \\ Michael Stiefel ${ }^{2}$ \\ Matthias Alexander Zingg ${ }^{2}$ \\ Thomas Rosemann² \\ Christoph Alexander Rüst ${ }^{2}$ \\ 'Gesundheitszentrum St Gallen, \\ St Gallen, ${ }^{2}$ Institute of Primary \\ Care, University of Zurich, Zurich, \\ Switzerland
}

Objective: This narrative review summarizes findings for Ironman triathlon performance and intends to determine potential predictor variables for Ironman race performance in female and male triathletes.

Methods: A literature search was performed in PubMed using the terms "Ironman", "triathlon", and "performance". All resulting articles were searched for related citations.

Results: Age, previous experience, sex, training, origin, anthropometric and physiological characteristics, pacing, and performance in split disciplines were predictive. Differences exist between the sexes for anthropometric characteristics. The most important predictive variables for a fast Ironman race time were age of 30-35 years (women and men), a fast personal best time in Olympic distance triathlon (women and men), a fast personal best time in marathon (women and men), high volume and high speed in training where high volume was more important than high speed (women and men), low body fat, low skin-fold thicknesses and low circumference of upper arm (only men), and origin from the United States of America (women and men).

Conclusion: These findings may help athletes and coaches to plan an Ironman triathlon career. Age and previous experience are important to find the right point in the life of a triathlete to switch from the shorter triathlon distances to the Ironman distance. Future studies need to correlate physiological characteristics such as maximum oxygen uptake with Ironman race time to investigate their potential predictive value and to investigate socio-economic aspects in Ironman triathlon.

Keywords: swimming, cycling, running, age, body fat, sex

\section{Introduction}

A triathlon is a multistage competition involving the completion of three continuous and sequential endurance disciplines swimming, cycling, and running. The "Ironman Hawaii", covering $3.8 \mathrm{~km}$ swimming, $180 \mathrm{~km}$ cycling, and $42.195 \mathrm{~km}$ running, is the best-known long-distance triathlon. The "Ironman Hawaii" triathlon is the World Championship of Ironman triathlon races ${ }^{1}$ and is considered as one of the 12 toughest endurance races in the world. ${ }^{2}$ Each year, tens of thousands of athletes try to qualify in Ironman qualifier races all around the world to finally qualify for "Ironman Hawaii"., 3,4 In both the qualifiers and in "Ironman Hawaii", athletes are ranked either as pro athletes or age group athletes. ${ }^{3,4}$

The intention of this narrative review is to give an overview of recent findings of potential predictor variables for a successful outcome in an Ironman triathlon. The findings might help athletes and coaches to plan a future career as Ironman triathlete. A literature search was performed using the data base PubMed ${ }^{5}$ using the terms
Correspondence: Beat Knechtle Gesundheitszentrum St Gallen, Vadianstrasse 26, 900I St Gallen, Switzerland

$\mathrm{Tel}+4 \mid$ II 2268282

Fax +4I 7| 2268272

Email beat.knechtle@hispeed.ch 
"Ironman" and "triathlon" leading to 176 results. We then refined the search using the terms "Ironman", "triathlon", and "performance". This search resulted in 76 publications. We then refined to studies investigating potential associations of selected variables with performance in Ironman triathlon races. The references of the selected studies were checked for further studies. We found age (ten publications), previous experience (six publications), sex (six publications), training (nine publications), anthropometry (18 publications), and origin (three publications) as potential predictors for Ironman race time. From a practical point of view, we further looked for publications investigating results of laboratory and blood analyses with practical relevance for athletes and coaches. We defined practical relevance as the fact that blood results could be replicated by a primary care physician, and the primary care physician would be able to explain his or her athlete the relevance of the blood results to change life and/ or training. A further search using the term "long-distance triathlon" resulted in the same references.

\section{Predictive characteristics}

\section{Age}

Age has been shown as an important predictor variable in both Half-Ironman ${ }^{6}$ and Ironman ${ }^{7-9}$ triathlon performance. The age of peak triathlon performance was higher in longer race distances in both women and men. For elite athletes competing in Olympic distance, Half-Ironman, and Ironman races at a high level, the ages of peak triathlon performance were $27.1 \pm 4.9,28.0 \pm 3.8$, and $35.1 \pm 3.6$ years, respectively, for men, and $26.6 \pm 4.4,31.6 \pm 3.4$, and $34.4 \pm 4.4$ years, respectively, for women. ${ }^{10}$ In Half-Ironman, women achieved their best race performances at the age of 25-39 years, whereas men attained their fastest race times between 18 and 39 years. ${ }^{11}$ Future studies would need to define more precisely the age of the best Half-Ironman performance. For Ironman, the age of the best triathlon performance was at the age of 30-35 years. ${ }^{11-13}$ Both women and men peaked at a similar age of 32-33 years with no sex difference. ${ }^{12,13}$

In a longitudinal study investigating the age of peak athletic performance in "Ironman Switzerland" from 1995 to 2010, no sex difference in the age of the best Ironman performance was found. ${ }^{12}$ For split disciplines, the ages of the best swimming, cycling, and running performance were different between women and men. ${ }^{13}$ In athletes competing in the qualifier "Ironman Switzerland" between 1995 and 2011, the best male swimmers were significantly younger (29 \pm 3 years) than the best male runners ( $35 \pm 5$ years). ${ }^{13}$ For women, the ages of peak split performances were not significantly different between the three disciplines. ${ }^{13}$ When the ages of the top ten finishers for all qualifier races for "Ironman Hawaii" and "Ironman Hawaii" itself were determined in 2010 , the age of peak Ironman triathlon performance was $32.2 \pm 1.5$ years for men and 33.0 \pm 1.6 years for women with no sex difference. ${ }^{12}$

However, the age of the annual ten fastest triathletes increased across the last 30 years in "Ironman Hawaii". ${ }^{14}$ This is in accordance with relative improvements of Ironman performances in older age groups. ${ }^{8,15}$ The mean age of the annual ten fastest finishers increased nonlinearly (ie, polynomial fifth degree) in women (Figure 1) and in men (Figure 2). Race times of the annual ten fastest decreased nonlinearly (ie, polynomial fifth degree) in women (Figure 3)

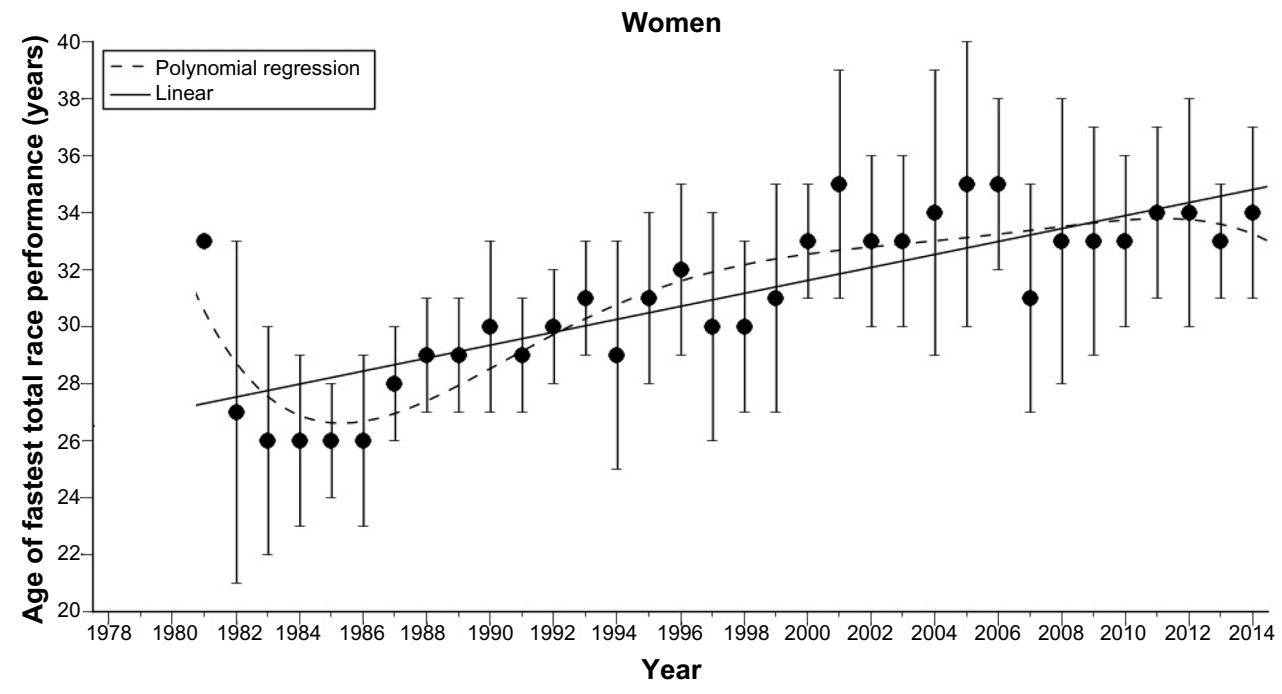

Figure I Age (years) of the annual ten fastest women in Ironman Hawaii from 1978 to 2014. Note: The results are presented as mean \pm SD. 


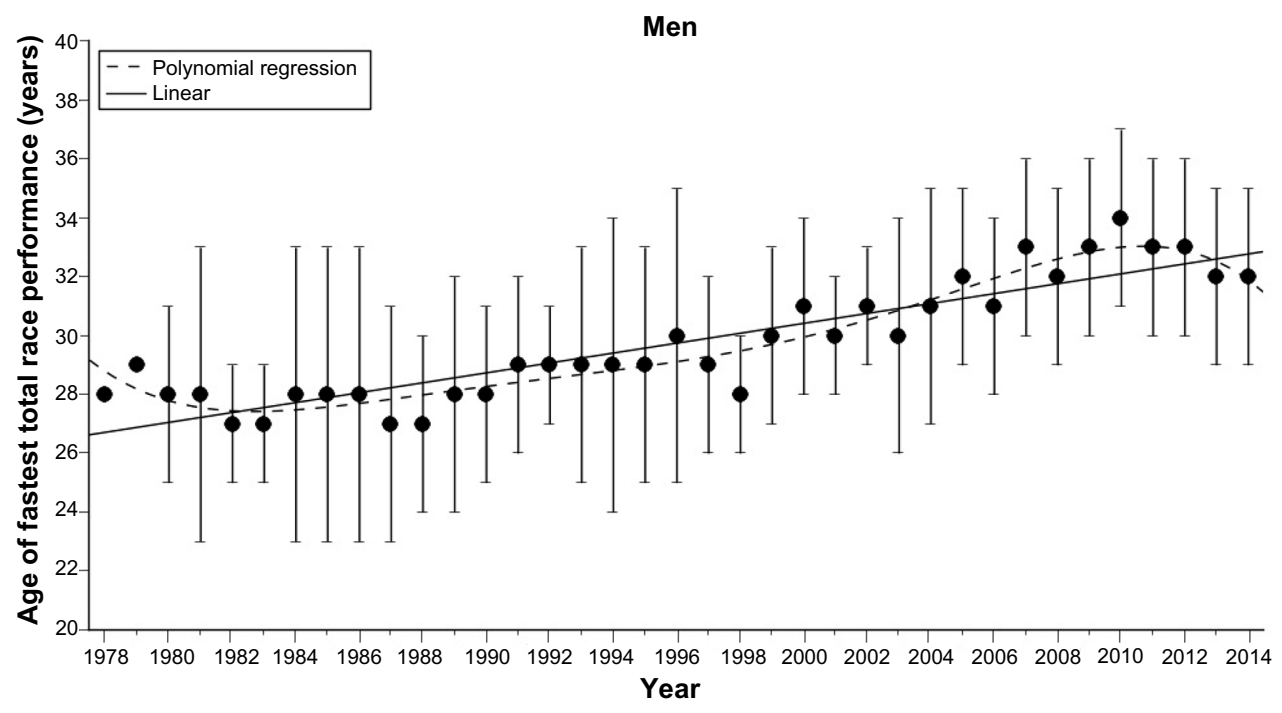

Figure 2 Age (years) of the annual ten fastest men in Ironman Hawaii from 1978 to 2014.

Note: The results are presented as mean \pm SD.

and men (Figure 4). Considering male winners in "Ironman Hawaii" in the last 11 years from 2004 to 2014, their mean age was 33.2 \pm 3.4 years (Table 1). For female winners, the mean age was identical at $33.4 \pm 3.0$ years (Table 2 ).

The age-related decline in triathlon performance depended on the race distance. With advancing age, the performance decline was less pronounced in Olympic distance triathlon compared with Ironman distance triathlon in cycling ( $>55$ years) and running ( $>50$ years), respectively. ${ }^{7}$ In contrast, an age-related decline in swimming performance was independent of the race distance. ${ }^{7}$ The age-related decline in triathlon performance was specific to each split discipline, with cycling showing a lower decline in performance with increasing age compared with swimming and running. ${ }^{?}$

Age was an important predictive variable for qualifying for "Ironman Hawaii" since there were differences in terms of participation and performance for athletes in different age groups between "Ironman Hawaii" and its qualifier races. ${ }^{4}$ In "Ironman Hawaii", men and athletes aged 25-49 years were underrepresented compared to the qualifiers. ${ }^{4}$ Similarly, women and athletes younger than 25 years and older than 50 years were relatively over-represented. Therefore, men and younger ( $<25$ years) and older ( $>50$ years) athletes had a lower chance to qualify for "Ironman Hawaii" than women. ${ }^{4}$

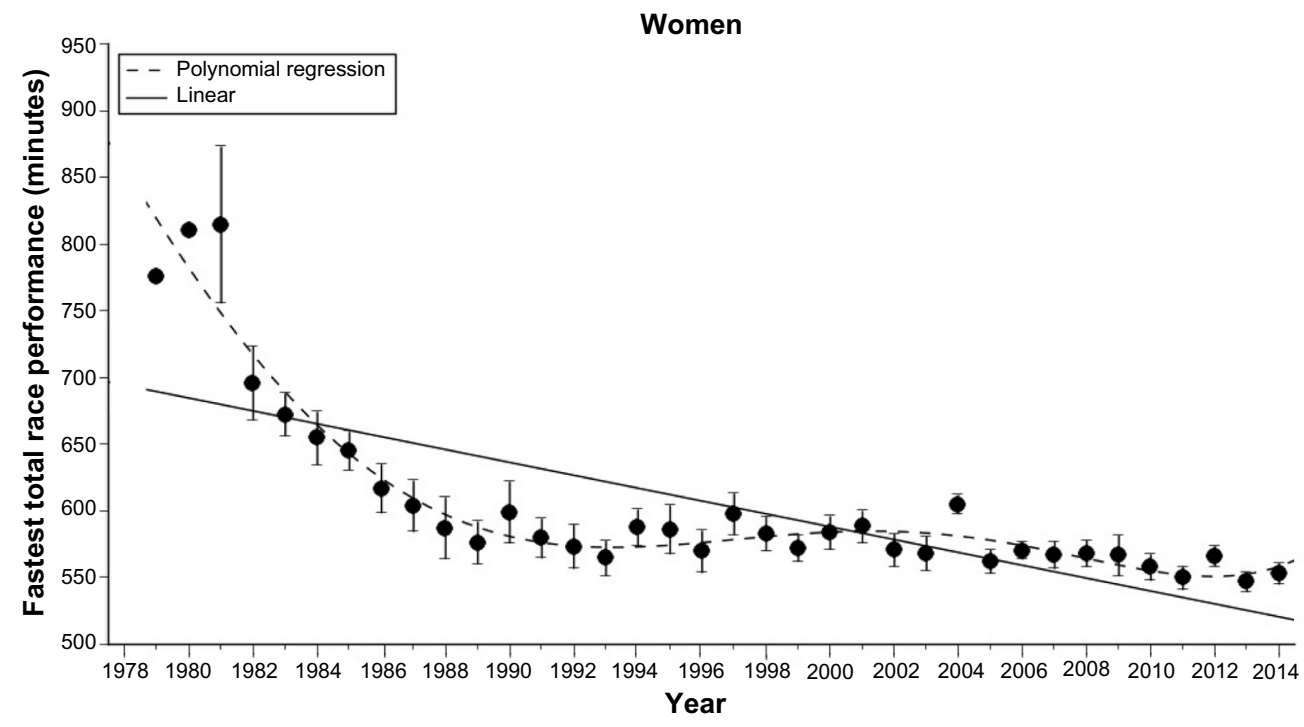

Figure 3 Overall race time of the annual ten fastest women in Ironman Hawaii from 1978 to 2014 . Note: The results are presented as mean \pm SD. 


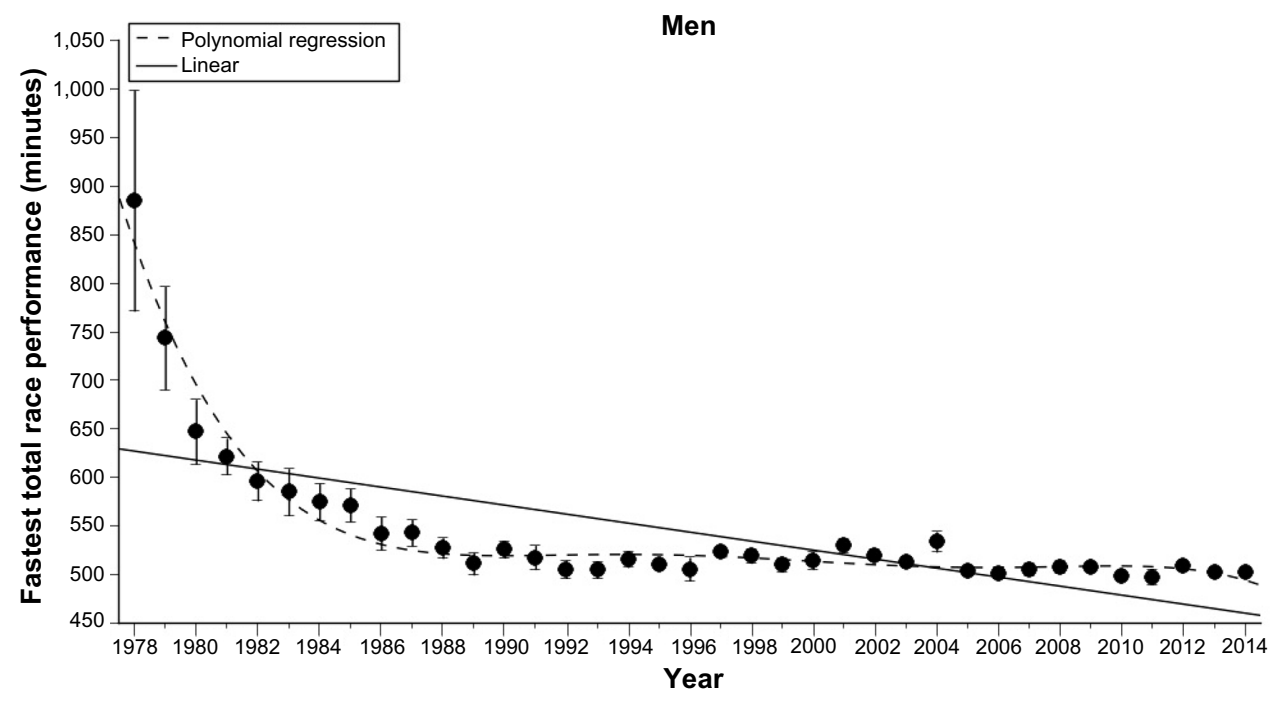

Figure 4 Overall race time of the annual ten fastest men in Ironman Hawaii from 1978 to 2014.

Note: The results are presented as mean \pm SD.

\section{Previous experience}

The number of completed previous triathlon $\operatorname{races}^{6}$ and the personal best times in Ironman triathlon and in shorter races such as Olympic distance triathlon ${ }^{16-20}$ were highly predictive for a fast Ironman race time. In Half-Ironman, faster athletes had completed more Half-Ironman races than slower athletes. ${ }^{6}$ The personal best time in an Olympic distance triathlon has been found as a strong predictor variable for a fast Ironman race time in female ${ }^{16,20}$ and male ${ }^{16,17,19}$ Ironman triathletes.

The personal best marathon time has also been found a strong predictor variable for Ironman race time in both female ${ }^{20}$ and male ${ }^{19}$ triathletes. The personal best marathon time was significantly and positively related to the run split time in an Ironman triathlon for male triathletes. ${ }^{17}$ The combination of both a fast personal best time in marathon and a fast personal best time in Olympic distance triathlon was highly predictive for a fast Ironman race time. In male Ironman triathletes, running speed during training, personal best marathon time, and personal best time in an Olympic distance triathlon were related to Ironman race time and explained $64 \%$ of the variance for Ironman race time. ${ }^{17}$ Previous best performances in Olympic distance triathlon coupled with weekly cycling distances and longest training rides could partially predict ( $57 \%$ of the variance) overall Ironman triathlon performance. ${ }^{16}$ Additionally, the personal best time in Ironman triathlons was a strong predictor variable for Ironman race time in both female and male triathletes. ${ }^{18}$ For athletes and coaches, Ironman race time (minutes) might be partially predicted in men using the formula: Ironman race time $($ minutes $)=152.1$ minutes $+1.964 \times$ personal best time in Olympic distance triathlon (minutes) $+1.332 \times$ personal best time in a marathon (minutes). ${ }^{19}$ For women, the equation is Ironman race time $($ minutes $)=186.3$ minutes $+1.595 \times$ personal best time in Olympic distance triathlon (minutes) + $1.318 \times$ personal best time in a marathon (minutes) ${ }^{20}$

Considering male (Table 1) and female (Table 2) winners in "Ironman Hawaii" in the last 11 years from 2004 to 2014, all female and male winners won at least one Ironman triathlon prior to their victory in "Ironman Hawaii". Female winners (Table 2) seemed to have larger previous experience in Ironman races and shorter races than male winners (Table 1). In women, several athletes (eg, Mirinda Carfrae, Leanda Cave, Chrissie Wellington, Michellie Jones, and Natascha Badmann) finished in Olympic Games, in a World Championship or in a Continental Championship within the first three (Table 2). The Swiss triathlete Nicola Spirig (born 1982) is an outstanding example showing that previous experience in Olympic distance triathlon and marathon road running are important predictors for a fast Ironman race time. ${ }^{21}$ Nicola Spirig won in November 2014 at the age of 32 years "Ironman Cozumel" in Mexico while competing in her first Ironman ever. A few months before this victory, Nicola Spirig came second in the Swiss national championship in marathon road running and 24th in the European championship in marathon road running. In spring 2015, she came second in the "Zürich Marathon". ${ }^{21}$ In 2012, Nicola Spirig was Olympic champion in Olympic distance triathlon in the London Olympic Games at the age of 30 years. In 2009, 2010, 2011, and 2014, she was European Champion in Olympic distance triathlon. And during her career, she won several Half-Ironman triathlons. ${ }^{21}$ 
Table I Male winners in Ironman Hawaii in the last eleven editions from 2004 to 2014 with their age at the victory and the previous experience (ie, top three rankings Ironman and shorter races at top level) before their victory in Ironman Hawaii

\begin{tabular}{|c|c|c|c|c|c|}
\hline Year & $\begin{array}{l}\text { Name } \\
\text { (age, years) }\end{array}$ & $\begin{array}{l}\text { First place } \\
\text { (age, years) }\end{array}$ & $\begin{array}{l}\text { Second place } \\
\text { (age, years) }\end{array}$ & $\begin{array}{l}\text { Third place } \\
\text { (age, years) }\end{array}$ & Reference \\
\hline \multirow[t]{4}{*}{2014} & Sebastian Kienle (30) & Ironman Frankfurt 2014 (30) & Ironman Frankfurt 2012 (28) & Ironman Hawaii & 83 \\
\hline & & Half-Ironman Las Vegas 2013 (29) & Ironman Roth 20II (27) & $2013(29)$ & \\
\hline & & Half-Ironman Las Vegas 2012 (28) & Ironman Roth 2010 (26) & & \\
\hline & & Half-Ironman Wiesbaden 2009 (25) & Half-Ironman Wiesbaden 2010 (26) & & \\
\hline \multirow[t]{6}{*}{2013} & Frederik Van & Ironman Nice 2013 (34) & Ironman Nice 2010 (3I) & Ironman Hawaii & 84 \\
\hline & Lierde (34) & Ironman Abu Dhabi 2013 (34) & Ironman Taupo 2008 (28) & $2012(33)$ & \\
\hline & & Ironman Nice 2012 (33) & & Ironman Melbourne & \\
\hline & & Ironman Nice 20I I (32) & & $2012(33)$ & \\
\hline & & Ironman Abu Dhabi 20II (32) & & & \\
\hline & & Half-Ironman Brasschaat 2007 (27) & & & \\
\hline \multirow[t]{7}{*}{2012} & Pete Jacobs (30) & Ironman Australia 201 I (29) & Ironman Lake Placid 2012 (30) & Ironman Western & 85 \\
\hline & & & Ironman Hawaii 20II (29) & Australia $2010(28)$ & \\
\hline & & & Ironman Roth 2009 (27) & Ironman Roth & \\
\hline & & & Ironman Australia 2009 (27) & $2007(25)$ & \\
\hline & & & Ironman Roth 2008 (26) & & \\
\hline & & & Ironman Wisconsin 2005 (23) & & \\
\hline & & & Ironman Western Australia 2004 (2I) & & \\
\hline \multirow[t]{6}{*}{2011} & Craig Alexander (38) & Half-Ironman Las Vegas 20I I (38) & Ironman Hawaii (WM) 2007 (34) & Ironman Australia & 86 \\
\hline & & Ironman Coeur d'Alene 20II (38) & & $2007(34)$ & \\
\hline & & Ironman Hawaii 2009 (36) & & & \\
\hline & & Ironman Hawaii 2008 (35) & & & \\
\hline & & Half-Ironman Clearwater & & & \\
\hline & & $2006(33)$ & & & \\
\hline \multirow[t]{11}{*}{2010} & Chris McCormack (37) & Ironman Frankfurt 2008 (35) & Ironman Hawaii 2006 (33) & Ironman Frankfurt & 87 \\
\hline & & Ironman Roth 2007 (34) & Ironman Roth 2003 (30) & $2010(37)$ & \\
\hline & & Ironman Hawaii 2007 (34) & & Ironman Frankfurt & \\
\hline & & Ironman Roth 2006 (33) & & $2009(36)$ & \\
\hline & & Ironman Australia 2006 (33) & & & \\
\hline & & Ironman Roth 2005 (32) & & & \\
\hline & & Ironman Australia 2005 (32) & & & \\
\hline & & Ironman Roth 2004 (3I) & & & \\
\hline & & Ironman Australia 2004 (3I) & & & \\
\hline & & Ironman Australia 2003 (30) & & & \\
\hline & & Ironman Australia 2002 (29) & & & \\
\hline \multirow[t]{3}{*}{2009} & Craig Alexander (36) & Ironman Hawaii 2008 (35) & Ironman Hawaii (WM) 2007 (34) & Ironman Australia & 86 \\
\hline & & Half-Ironman Clearwater & & $2007(34)$ & \\
\hline & & $2006(33)$ & & & \\
\hline \multirow[t]{2}{*}{2008} & Craig Alexander (35) & Half-Ironman Clearwater & Ironman Hawaii (WM) 2007 (34) & Ironman Australia & 86 \\
\hline & & $2006(33)$ & & $2007(34)$ & \\
\hline \multirow[t]{8}{*}{2007} & Chris McCormack (34) & Ironman Roth 2006 (33) & Ironman Hawaii 2006 (33) & & 87 \\
\hline & & Ironman Australia 2006 (33) & Ironman Roth 2003 (30) & & \\
\hline & & Ironman Roth 2005 (32) & & & \\
\hline & & Ironman Australia 2005 (32) & & & \\
\hline & & Ironman Roth 2004 (3I) & & & \\
\hline & & Ironman Australia 2004 (3I) & & & \\
\hline & & Ironman Australia 2003 (30) & & & \\
\hline & & Ironman Australia 2002 (29) & & & \\
\hline \multirow[t]{4}{*}{2006} & Normann Stadler (33) & Ironman Frankfurt 2005 (32) & Ironman Zürich 2004 (3I) & & 88 \\
\hline & & Ironman Hawaii 2004 (3I) & Ironman Hawaii 2000 (27) & & \\
\hline & & Ironman Australia 200I(28) & & & \\
\hline & & Ironman Australia 2000 (27) & & & \\
\hline \multirow[t]{2}{*}{2005} & Faris Al-Sultan (27) & Ironman Arizona 2005 (27) & Ironman Roth 2004 (26) & Ironman Hawaii & 89 \\
\hline & & & Ironman Brasil 200I (23) & $2004(26)$ & \\
\hline \multirow[t]{2}{*}{2004} & Normann Stadler (3I) & Ironman Australia $200 \mathrm{I}(28)$ & Ironman Zürich 2004 (3I) & & 88 \\
\hline & & Ironman Australia 2000 (27) & Ironman Hawaii 2000 (27) & & \\
\hline
\end{tabular}

Abbreviation: WM, World Championship. 
Table 2 Female winners in Ironman Hawaii in the last eleven editions from 2004 to 2014 with their age at the victory and the previous experience (ie, top three rankings Ironman and shorter races at top level) before their victory in Ironman Hawaii

\begin{tabular}{|c|c|c|c|c|c|}
\hline Year & $\begin{array}{l}\text { Name } \\
\text { (age, years) }\end{array}$ & $\begin{array}{l}\text { First place } \\
\text { (age, years) }\end{array}$ & $\begin{array}{l}\text { Second place } \\
\text { (age, years) }\end{array}$ & $\begin{array}{l}\text { Third place } \\
\text { (age, years) }\end{array}$ & Reference \\
\hline 2014 & $\begin{array}{l}\text { Mirinda } \\
\text { Carfrae (33) }\end{array}$ & $\begin{array}{l}\text { Ironman Roth } 20 \text { I4 (33) } \\
\text { Ironman Hawaii } 2013(32) \\
\text { Ironman Hawaii } 2010(29) \\
\text { Half-Ironman Clearwater } 2007(26)\end{array}$ & $\begin{array}{l}\text { Ironman Florida } 20 \text { I2 (3I) } \\
\text { Ironman Hawaii } 20 \text { I I (30) } \\
\text { Ironman New Zealand 20I I (30) } \\
\text { Ironman Hawaii } 2009 \text { (28) } \\
\text { ITU World Long Distance Triathlon } \\
\text { Championship } 2005 \text { (24) } \\
\text { ITU Triathlon World Championship } \\
\text { U23 } 2003 \text { (22) }\end{array}$ & $\begin{array}{l}\text { Ironman Hawaii } \\
20 I 2(3 I) \\
\text { Ironman Melbourne } \\
20 I 2(3 I) \\
\text { Half-Ironman } \\
\text { Clearwater } 2006(25)\end{array}$ & 90 \\
\hline 2013 & $\begin{array}{l}\text { Mirinda } \\
\text { Carfrae (32) }\end{array}$ & $\begin{array}{l}\text { Ironman Hawaii } 2010 \text { (29) } \\
\text { Half-Ironman Clearwater } 2007 \text { (26) }\end{array}$ & $\begin{array}{l}\text { Ironman Florida } 20 \text { I2 (3I) } \\
\text { Ironman Hawaii } 20 \text { II (30) } \\
\text { Ironman New Zealand 20I I (30) } \\
\text { Ironman Hawaii } 2009 \text { (28) } \\
\text { ITU World Long Distance Triathlon } \\
\text { Championship } 2005 \text { (24) } \\
\text { ITU Triathlon World Championship } \\
\text { U23 } 2003 \text { (22) }\end{array}$ & $\begin{array}{l}\text { Ironman Hawaii } \\
20 I 2(3 I) \\
\text { Ironman Melbourne } \\
20 I 2(3 I) \\
\text { Half-Ironman } \\
\text { Clearwater } 2006(25)\end{array}$ & 90 \\
\hline 2012 & $\begin{array}{l}\text { Leanda Cave } \\
\text { (34) }\end{array}$ & $\begin{array}{l}\text { Half-Ironman Las Vegas } 2012 \text { (34) } \\
\text { Ironman Arizona } 2011 \text { ( } 33 \text { ) } \\
\text { ITU Triathlon Long Distance World } \\
\text { Championships } 2007 \text { (29) } \\
\text { ITU Triathlon Short Distance World } \\
\text { Championships } 2002 \text { (24) } \\
\text { ETU European Triathlon Short } \\
\text { Distance Championships U23 200I (23) }\end{array}$ & $\begin{array}{l}\text { ITU Triathlon Long Distance World } \\
\text { Championships } 201 \mathrm{I} \text { ( } 33 \text { ) } \\
\text { Ironman Abu Dhabi } 20 \text { I0 (32) } \\
\text { Half-Ironman Clearwater } 2010 \text { (32) } \\
\text { Ironman Arizona } 2008 \text { (30) } \\
\text { ETU European Triathlon Short } \\
\text { Distance Championships } 2002 \text { (24) }\end{array}$ & $\begin{array}{l}\text { Ironman Hawaii } \\
2011 \text { ( } 33) \\
\text { Ironman Arizona } \\
201 \text { I (33) } \\
\text { Half-Ironman } \\
\text { Clearwater } 2007 \text { (29) }\end{array}$ & 91 \\
\hline 2011 & $\begin{array}{l}\text { Chrissie } \\
\text { Wellington } \\
(34)\end{array}$ & $\begin{array}{l}\text { Ironman Roth } 20 \text { II (34) } \\
\text { Ironman South Africa 20I I (34) } \\
\text { Ironman Arizona } 20 \text { I0 (33) } \\
\text { Ironman Roth } 2010(33) \\
\text { Ironman Hawaii } 2009(32) \\
\text { Ironman Roth } 2009(32) \\
\text { Ironman Australia } 2009(32) \\
\text { Ironman Hawaii } 2008(3 \text { I) } \\
\text { ITU Triathlon Long Distance World } \\
\text { Championships } 2008(3 \text { I) } \\
\text { Ironman Germany } 2008(3 \text { I) } \\
\text { Ironman Australia } 2008(3 \text { I) } \\
\text { Ironman Hawaii } 2007(30) \\
\text { Ironman Korea } 2007(30) \\
\text { ITU Triathlon World Championship } \\
2006 \text { (29) }\end{array}$ & & & 92 \\
\hline 2010 & $\begin{array}{l}\text { Mirinda } \\
\text { Carfrae (29) }\end{array}$ & Half-Ironman Clearwater 2007 (26) & $\begin{array}{l}\text { Ironman Hawaii } 2009(28) \\
\text { ITU World Long Distance Triathlon } \\
\text { Championship } 2005 \text { (24) } \\
\text { ITU Triathlon World Championship } \\
\text { U23 } 2003 \text { (22) }\end{array}$ & $\begin{array}{l}\text { Half-Ironman } \\
\text { Clearwater } 2006 \text { (25) }\end{array}$ & 90 \\
\hline 2009 & $\begin{array}{l}\text { Chrissie } \\
\text { Wellington } \\
(32)\end{array}$ & $\begin{array}{l}\text { Ironman Roth } 2009(32) \\
\text { Ironman Australia } 2009(32) \\
\text { Ironman Hawaii } 2008(3 \mathrm{I}) \\
\text { ITU Triathlon Long Distance World } \\
\text { Championships } 2008(3 \mathrm{I}) \\
\text { Ironman Germany } 2008(3 \mathrm{I}) \\
\text { Ironman Australia } 2008(3 \mathrm{I}) \\
\text { Ironman Hawaii } 2007(30) \\
\text { Ironman Korea } 2007(30) \\
\text { ITU Triathlon World Championship } \\
2006(29)\end{array}$ & & & 92 \\
\hline
\end{tabular}


Table 2 (Continued)

\begin{tabular}{|c|c|c|c|c|c|}
\hline Year & $\begin{array}{l}\text { Name } \\
\text { (age, years) }\end{array}$ & $\begin{array}{l}\text { First place } \\
\text { (age, years) }\end{array}$ & $\begin{array}{l}\text { Second place } \\
\text { (age, years) }\end{array}$ & Third place (age, years) & Reference \\
\hline 2008 & $\begin{array}{l}\text { Chrissie } \\
\text { Wellington } \\
(3 \mathrm{I})\end{array}$ & $\begin{array}{l}\text { ITU Triathlon Long Distance World } \\
\text { Championships } 2008(3 \mathrm{I}) \\
\text { Ironman Germany } 2008(3 \mathrm{I}) \\
\text { Ironman Australia } 2008(3 \mathrm{I}) \\
\text { Ironman Hawaii } 2007(30) \\
\text { Ironman Korea } 2007(30) \\
\text { ITU Triathlon World Championship } \\
2006(29)\end{array}$ & & & 92 \\
\hline 2007 & $\begin{array}{l}\text { Chrissie } \\
\text { Wellington } \\
(30)\end{array}$ & $\begin{array}{l}\text { Ironman Korea } 2007 \text { (30) } \\
\text { ITU Triathlon World Championship } \\
2006 \text { (29) }\end{array}$ & & & 92 \\
\hline 2006 & $\begin{array}{l}\text { Michellie } \\
\text { Jones (37) }\end{array}$ & $\begin{array}{l}\text { Ironman Arizona } 2006 \text { (37) } \\
\text { Ironman Florida } 2004 \text { (35) } \\
\text { ITU Triathlon Short Distance World } \\
\text { Championships I } 993 \text { (24) } \\
\text { ITU Triathlon Short Distance World } \\
\text { Championships I } 992 \text { (23) }\end{array}$ & $\begin{array}{l}\text { Ironman Hawaii } 2005 \text { (36) } \\
\text { ITU Triathlon Short Distance World } \\
\text { Championships 200I (32) } \\
\text { Triathlon Olympic Games } \\
2000 \text { (3I) } \\
\text { ITU Triathlon Short Distance } \\
\text { World Championships } 1998 \text { (29) }\end{array}$ & $\begin{array}{l}\text { ITU Triathlon Short } \\
\text { Distance World } \\
\text { Championships } 2003 \text { (34) } \\
\text { ITU Triathlon Short } \\
\text { Distance World } \\
\text { Championships } 2000 \text { (3I) } \\
\text { ITU Triathlon Short } \\
\text { Distance World } \\
\text { Championships I997 (28) } \\
\text { ITU Triathlon Short } \\
\text { Distance World } \\
\text { Championships I99I (22) }\end{array}$ & 93 \\
\hline 2005 & $\begin{array}{l}\text { Natascha } \\
\text { Badmann (38) }\end{array}$ & $\begin{array}{l}\text { Ironman South Africa } 2005(38) \\
\text { Ironman Hawaii } 2004(37) \\
\text { Ironman Hawaii } 2002(35) \\
\text { Ironman Hawaii } 200 \text { I (34) } \\
\text { Ironman California } 200 \text { I (34) } \\
\text { Ironman Hawaii } 2000(33) \\
\text { Ironman Hawaii I998 (3I) } \\
\text { ETU European Triathlon Short } \\
\text { Distance Championship I997 (30) }\end{array}$ & $\begin{array}{l}\text { Ironman Hawaii } 2003 \text { (36) } \\
\text { ITU Triathlon Long Distance World } \\
\text { Championships } 2000 \text { (33) } \\
\text { Ironman Hawaii I } 996 \text { (29) } \\
\text { ETU European Triathlon Short } \\
\text { Distance Championship I995 (28) } \\
\text { ETU European Triathlon Short } \\
\text { Distance Championship I994 (27) }\end{array}$ & & 94 \\
\hline 2004 & $\begin{array}{l}\text { Natascha } \\
\text { Badmann (37) }\end{array}$ & $\begin{array}{l}\text { Ironman Hawaii } 2002(35) \\
\text { Ironman Hawaii } 200 \text { I (34) } \\
\text { Ironman California } 200 \text { I (34) } \\
\text { Ironman Hawaii } 2000(33) \\
\text { Ironman Hawaii I } 998(3 \text { I) } \\
\text { ETU European Triathlon Short } \\
\text { Distance Championship I997 (30) }\end{array}$ & $\begin{array}{l}\text { Ironman Hawaii } 2003 \text { (36) } \\
\text { ITU Triathlon Long Distance World } \\
\text { Championships } 2000(33) \\
\text { Ironman Hawaii I } 996(29) \\
\text { ETU European Triathlon Short } \\
\text { Distance Championship I995 (28) } \\
\text { ETU European Triathlon Short } \\
\text { Distance Championship I994 (27) }\end{array}$ & & 94 \\
\hline
\end{tabular}

Abbreviations: ETU, European Triathlon Union; ITU, International Triathlon Union.

\section{Sex}

Men were faster than women in triathlon races, ${ }^{7,22-24}$ but differences between the sexes exist for split performances. In athletes competing between 1988 and 2007 in "Ironman Hawaii", the sex difference in performance time was significantly smaller for swimming (9.8\%) compared with cycling $(12.7 \%)$ and running (13.3\%). ${ }^{7}$ During this period, the sex difference in swimming remained stable while it slightly increased in cycling but decreased in running. ${ }^{7}$ In "Ironman Switzerland" during the 1996-2010 period, the sex difference in running (18.2\%) was greater compared with swimming $(14.0 \%)$ and cycling (13.2\%). There was a decrease in the sex difference in performance in the three disciplines and for overall race time. ${ }^{12}$ In Half-Ironman ${ }^{11}$ and Ironman ${ }^{23}$ triathlon races, the sex difference depended upon the age of the athletes. In "Ironman Hawaii", the sex difference for overall race time was stable until the age of $\sim 55$ years and increased thereafter. ${ }^{23}$

\section{Training}

Training characteristics showed an important influence on Ironman triathlon race time. ${ }^{17,18,25}$ Faster finishers (ie, race time faster than 10:30 h:min in "Ironman Hawaii") tended to average greater training volumes at faster paces than 
slower finishers. ${ }^{26}$ However, sex differences exist for training characteristics regarding their association with Ironman race time $^{18}$ although female and male Ironman triathletes show no differences between volume and speed in training. ${ }^{26} \mathrm{~A}$ summary of recent studies showed that weekly training hours in swimming, cycling, and running were similar between female and male Ironman triathletes (Table 3). However, men were training more kilometers and at a higher speed compared with women (Table 3).

This results in different associations between training characteristics and both split and overall race times. Overall weekly training hours were related to Ironman race performance in women ${ }^{18}$ but not in men. ${ }^{27}$ Running speed during training was related to marathon split time in female Ironman triathletes ${ }^{28}$ and to overall race time in male Ironman triathletes. ${ }^{17}$ In male Ironman triathletes, weekly swimming kilometers were related to the swim split time ${ }^{29}$ and speed in cycling training was related to split time in cycling in the Ironman. ${ }^{29-31}$ In female Ironman triathletes, weekly cycling kilometers were related to overall race time. ${ }^{20}$ Considering swimming, no associations were reported for female Ironman triathletes.

The intensity during training also influences Ironman race time where a lower training intensity was associated with faster race times. ${ }^{32}$ Ironman triathletes trained mainly at a low intensity (ie, zone 1, low intensity, < aerobic threshold), whereas the Ironman race was primarily performed at the intensity in zone 2 (ie, moderate intensity, between aerobic and anaerobic threshold). ${ }^{32}$ There was a significant inverse correlation between both total training time and training time in zone 1 and Ironman race time. ${ }^{32}$ In contrast, a moderate and positive correlation between total training time in zone 2 and Ironman race time and a strong positive correlation between percentage of total training time in zone 2 and
Ironman race time was reported..$^{32}$ In other terms, a too high intensity during Ironman training might be a disadvantage for a fast race performance.

\section{Anthropometric characteristics}

Anthropometric characteristics are of high importance for Ironman race performance. ${ }^{18,33-35}$ Kandel et al showed that somatotype was a strong predictor variable of Ironman race performance in male athletes competing in "Ironman Switzerland" where the endomorphy component (ie, having a rounded, stocky body structure with a tendency to obesity) was the most important predictor. ${ }^{33}$ A reduction in endomorphy and an increase in ectomorphy (ie, having a light, slender body structure) leads to significant and substantial improvements in Ironman race performance. ${ }^{33}$ Lower body mass, lower body mass index, and lower body fat were associated with both a faster Ironman race and a faster run split. ${ }^{34}$

Among the anthropometric characteristics, body fat was the most important predictor variable for Half-Ironman ${ }^{6}$ and Ironman ${ }^{27,29-31,34,36,37}$ race performance. In Half-Ironman, faster athletes had lower body fat than slower ones. ${ }^{6}$ Percent body fat was differentially related to split and overall performance regarding the sex of the athletes. ${ }^{18}$ In male Ironman triathletes, percent body fat was inversely related to overall race time $\mathrm{e}^{18,27,37}$ and in the split times in cycling ${ }^{37}$ and running. ${ }^{29}$ In female Ironman triathletes, however, percent body fat was not related to overall Ironman race time..$^{18,20,27}$

Skin-fold thicknesses were important for split and overall race time in Ironman triathlon. ${ }^{31}$ However, differences do exist between the sexes in skin-fold thicknesses. ${ }^{37}$ In male Ironman triathletes, the skin-fold thicknesses at abdominal and iliacal site were associated with race time. The abdominal and iliacal skin-fold thicknesses were related to cycling split times in the Ironman race. ${ }^{31}$ The sum of upper body skinfolds

Table 3 Weekly volume in hours $(\mathrm{h})$ and kilometers $(\mathrm{km})$ and speed in training $(\mathrm{km} / \mathrm{h})$ in female and male Ironman triathletes

\begin{tabular}{|c|c|c|c|c|c|c|c|c|c|c|c|}
\hline & \multirow{2}{*}{$\begin{array}{l}\text { Total } \\
\text { h }\end{array}$} & \multicolumn{3}{|c|}{ Swimming } & \multicolumn{3}{|c|}{ Cycling } & \multicolumn{3}{|c|}{ Running } & \multirow[t]{2}{*}{ Reference } \\
\hline & & h & km & km/h & h & km & km/h & h & km & km/h & \\
\hline \multirow[t]{6}{*}{ Men } & 13.5 & 2.4 & 6.4 & 3.0 & 7.3 & 206.0 & 29.0 & 3.7 & 41.0 & 11.7 & 36 \\
\hline & 14.7 & & 6.0 & 2.8 & & 189.6 & 28.1 & & 44.8 & 11.2 & 17 \\
\hline & 14.8 & 2.5 & 6.7 & 2.7 & 8.0 & 220.5 & 27.3 & 4.0 & 42.0 & 10.0 & 27 \\
\hline & 13.9 & 2.6 & 6.3 & 2.9 & 7.1 & 194.4 & 28.4 & 4.3 & 45.0 & 11.6 & 19 \\
\hline & & & 8.8 & & & 270.0 & & & 58.2 & & 16 \\
\hline & 13.9 & 2.5 & 6.2 & 2.9 & 7.1 & 194.0 & 28.4 & 4.3 & 45.0 & 11.5 & 34 \\
\hline Mean \pm SD & $14.2 \pm 0.7$ & $2.5 \pm 0.1$ & $6.8 \pm 1.1$ & $2.8 \pm 0.1$ & $7.5 \pm 0.5$ & $216.1 \pm 32.4$ & $28.2 \pm 0.7$ & $4.0 \pm 0.3$ & $46.2 \pm 6.9$ & $\mid \mathrm{I} .1 \pm 0.8$ & \\
\hline \multirow[t]{3}{*}{ Women } & 13.9 & 2.4 & 5.5 & 2.1 & 7.5 & 192.3 & 25.0 & 4.0 & 45.0 & 10.3 & 27 \\
\hline & & & 5.5 & & & 190.0 & & & 42.0 & & 39 \\
\hline & 14.1 & 2.8 & 6.2 & 2.8 & 7.4 & 196.6 & 26.0 & 4.1 & 41.0 & 10.7 & 31 \\
\hline Mean \pm SD & $|4.0 \pm 0|$. & $2.6 \pm 0.3$ & $5.4 \pm 0.4$ & $2.4 \pm 0.5$ & $7.4 \pm 0.1$ & $192.9 \pm 3.3$ & $25.5 \pm 0.7$ & $4.0 \pm 0.1$ & $42.7 \pm 2.1$ & $10.5 \pm 0.3$ & \\
\hline
\end{tabular}

Abbreviation: SD, standard deviation. 
and the sum of eight skinfolds were related to cycling speed in the cycle split and to overall race time. ${ }^{37}$ In females Ironman triathletes, however, none of the skinfold thicknesses showed an association with overall race time or speed in the split disciplines in the Ironman race. ${ }^{37}$

Limb circumferences were related to Ironman split times. In male Ironman triathletes, lower circumferences of upper arm and thigh were related to a faster run split. ${ }^{34}$ Upper arm circumference was also related to overall race time. ${ }^{30}$

A further aspect regarding anthropometry is the loss in body mass during a triathlon race such as a Half-Ironman. ${ }^{38}$ For example, in a Half-Ironman, the change in body mass correlated positively with race time where greater reductions in body mass were found in faster athletes. ${ }^{38}$ In Ironman triathletes, however, changes in body mass differ between the sexes. ${ }^{39,40}$ While male Ironman triathletes lost body mass during the race, ${ }^{40,41}$ body mass remained unchanged in female Ironman triathletes. ${ }^{39}$ The loss in body mass in male Ironman triathletes was mainly due to a loss in skeletal muscle mass due to glycogen depletion. ${ }^{41,42}$

\section{Origin of the athletes}

The "Ironman Hawaii" was invented in Hawaii, USA. ${ }^{43}$ Since its first edition, women and men from the USA dominated both participation and performance in "Ironman Hawaii". ${ }^{44}$ Between 1985 and 2012, most of the finishers originated from the USA (47.5\%) followed by athletes from Germany (11.7\%), Japan (7.9\%), Australia (6.7\%), Canada (5.2\%), Switzerland (2.9\%), France (2.3\%), Great Britain (2.0\%), New Zealand (1.9\%), and Austria (1.5\%). ${ }^{44}$ Regarding the fastest race times ever, the fastest women originated from the USA followed by Great Britain, and Switzerland. In men, the fastest finishers originated from the USA, Germany, and Australia. $^{44}$

For the qualifiers for "Ironman Hawaii", however, differences do exist when all finishers in 2010 in both "Ironman Hawaii" and in the qualifier races were analyzed. ${ }^{3}$ Considering sex, a higher percentage of women (27.2\%) finished in "Ironman Hawaii" compared with the qualifier races where only $18.9 \%$ of women finished. Considering men, however, a higher percentage $(81.1 \%)$ finished in the qualifiers compared with "Ironman Hawaii" where only $72.8 \%$ finished. ${ }^{3}$ Most of the finishers originated from the USA in both "Ironman Hawaii" and its qualifier races. Behind US-athletes, competitors from Germany and Canada finished also very frequently. ${ }^{3}$ When sex and origin was investigated, there were also differences in "Ironman Hawaii" and its qualifier races. In "Ironman Hawaii", the percentage of women was lower for US and Canadian athletes and higher for German athletes. For men, the percentage was higher for athletes from the USA and Germany but lower for athletes from Canada. ${ }^{3}$

Apart from the differences in participation and finishers trends, there were also differences in performance trends between "Ironman Hawaii" and its qualifier races. Overall races times were faster in both women and men in the qualifier races compared with "Ironman Hawaii". ${ }^{3}$ In the qualifier races, the fastest women originated from the USA, Germany, and Switzerland. In "Ironman Hawaii", however, women from the USA, Australia, and Germany achieved the fastest race times. ${ }^{3}$ Women from Germany, Canada, Switzerland, and New Zealand competed faster in the qualifier races than in "Ironman Hawaii". ${ }^{3}$ For men, athletes from Germany, Australia, and Great Britain obtained the fastest race times in the qualifier races. ${ }^{3}$ In "Ironman Hawaii", US American, German, and Australian finishers were the fastest. ${ }^{3}$ Men from France, Great Britain, Switzerland, and Canada were faster in the qualifier races compared with "Ironman Hawaii". ${ }^{3}$ Most probably the qualifying system for "Ironman Hawaii" is not respecting the different nationalities of the athletes.

\section{Physiological characteristics}

Elite triathletes have high values for maximum oxygen uptake $\left(\mathrm{VO}_{2} \max \right){ }^{45}$ Although $\mathrm{VO}_{2} \max$ is a predictor of performance in triathletes of mixed abilities, it cannot be used to predict performance within homogenous groups of elite performers. ${ }^{45}$ Elite triathletes have significantly higher $\mathrm{VO}_{2}$ max values than subelite triathletes, and high $\mathrm{VO}_{2} \max$ levels are required to succeed in triathlons. ${ }^{45} \mathrm{VO}_{2} \max$ was higher in male compared with female Ironman triathletes. ${ }^{46}$ $\mathrm{VO}_{2}$ max at maximal exercise was, for males and females, respectively, $68.8 \mathrm{~mL} \mathrm{~kg}^{-1} \mathrm{~min}^{-1}, 65.9 \mathrm{~mL} \mathrm{~kg}^{-1} \mathrm{~min}^{-1}$ on the treadmill, $66.7 \mathrm{~mL} \mathrm{~kg}^{-1} \mathrm{~min}^{-1}, 61.6 \mathrm{~mL} \mathrm{~kg}^{-1} \mathrm{~min}^{-1}$ on the cycle ergometer, and $49.1 \mathrm{~mL} \mathrm{~kg}^{-1} \mathrm{~min}^{-1}, 39.7 \mathrm{~mL} \mathrm{~kg}^{-1} \mathrm{~min}^{-1}$ on the arm ergometer. ${ }^{46}$ In contrast to elite Ironman triathletes, ${ }^{46}$ recreational Ironman triathletes have lower values for male $\left(58.1 \pm 8.6 \mathrm{~mL} \mathrm{~kg}{ }^{-1} \mathrm{~min}^{-1}\right)$ and female $\left(52.8 \pm 5.7 \mathrm{~mL} \mathrm{~kg}^{-1}\right.$ $\min ^{-1}$ ) Ironman triathletes. ${ }^{47}$

Laboratory results may be used to predict triathlon race performance. ${ }^{48-52}$ For Olympic distance triathlon, the five most significant predictors of triathlon performance were blood lactate measured during steady-state cycling at a workload of $4 \mathrm{~W} \mathrm{~kg}^{-1}$ body mass, blood lactate while running at 15 $\mathrm{km} /$ hour, peak sustained power output, peak treadmill running velocity, and $\mathrm{VO}_{2}$ peak during cycling. ${ }^{52}$ The intensity at anaerobic threshold has been shown to be too high for the 
required intensity during an Ironman triathlon, and $\mathrm{VO}_{2}$ values at the lactate (ie, exercise intensity at which lactate starts to accumulate in the blood) and ventilatory thresholds (ie, point during exercise at which pulmonary ventilation becomes disproportionately high with respect to oxygen consumption) were not highly related to bike finish time. ${ }^{49} \mathrm{VO}_{2}$ at anaerobic threshold, percentage of $\mathrm{VO}_{2} \max$ at anaerobic threshold, and peak power to body mass ratio were not related to Ironman race times. ${ }^{53}$ Regarding split disciplines, fractional utilization of peak $\mathrm{VO}_{2}\left(\%\right.$ peak $\left.\mathrm{VO}_{2}\right)$, heart rate, and $\%$ peak heart rate at thresholds were not related to bike finish time. ${ }^{51}$ Ironman triathletes cycled during the Ironman race at a heart rate intensity that approximated to heart rate at the ventilatory threshold but at a power output that was significantly below power output at the ventilatory threshold..$^{50}$

A long-distance triathlon such as a Half-Ironman also leads to changes in laboratory results. Puggina et al showed an increase in creatinine in the blood and an increased excretion of protein, erythrocytes and leucocytes in the urine. ${ }^{54}$ In male Ironman triathletes, seasonal relationships between hematology and lymphocyte function, independent of endurance training were found and possibly affecting performance. ${ }^{55}$ After an Ironman triathlon, liver enzymes were considerably increased and remained elevated for 5-6 days after the race. ${ }^{56}$ Similarly, serum glucose, glycerol, and nonesterified fatty acids were increased postrace. ${ }^{56}$

\section{Pacing and performance in split disciplines}

Pacing and the performances in the single split disciplines were also related to Ironman race performance. ${ }^{54,55}$ There were differences regarding the performance in the split disciplines swimming, cycling, and running. In long-distance triathlon, times spent during cycling and running during the race were significantly related to overall race time. However, swimming time was not related to overall race time. ${ }^{53}$ Pacing during a triathlon also has an influence on overall performance. ${ }^{57}$ During an Ironman triathlon, cycling and running pacing on downhill segments predicted relative overall race success. ${ }^{58}$

\section{Other influences on performance in Ironman and ultra-triathlon}

Apart from studies investigating associations with potential predictor variables with race times, also other aspects need to be addressed to explain the demands for athletes competing in these races.

\section{Aspects of fluid metabolism}

Several studies investigated fluid ${ }^{59-69}$ and energy ${ }^{69-71}$ intake in Ironman triathlon. For Ironman triathletes, dehydration has been reported as a cause of fatigue, and exercise-associated hyponatremia has been highlighted as a major concern during such races. ${ }^{72}$ Dehydration and electrolyte balance are important for a successful race. Dehydration is common and exercise-associated hyponatremia is the predominant electrolyte disturbance in the "Ironman Hawaii". ${ }^{73}$ An Ironman can lead to exercise-associated hyponatremia ${ }^{65,67,74}$ caused mainly by fluid overload ${ }^{65}$ as a consequence of excessive drinking. ${ }^{62,74}$ Especially women seem to be at high risk for complications due to exercise-associated hyponatremia. ${ }^{75,76}$ However, also in male Ironman triathletes, exercise-associated hyponatremia can lead to serious problems. ${ }^{77}$ Ad libitum fluid intake is the best way to maintain plasma sodium concentration in Ironman triathletes. ${ }^{78}$

Changes in body mass were related to changes in serum $\left[\mathrm{Na}^{+}\right]$in both female and male Ironman triathletes. ${ }^{60}$ Sodium ingestion during an Ironman was associated with a decrease in the extent of body mass loss. However, there is no evidence that sodium ingestion significantly influences changes in serum $\left[\mathrm{Na}^{+}\right]$during an Ironman ${ }^{68}$ most probably due to the fact that an Ironman is not leading to large sodium losses. ${ }^{65}$ However, slowing down during the marathon in an Ironman could be due to hyperthermia and a reduction in plasma sodium concentration. ${ }^{79}$ For a HalfIronman, however, oral salt supplementation improved performance. ${ }^{80}$ Additionally, oral salt supplementation was effective to lessen body mass loss and to increase serum electrolyte concentration. ${ }^{80}$

\section{Aspects of energy metabolism}

Several studies investigated energy ${ }^{69-71}$ intake in Ironman triathlon. Athletes competing in an Ironman triathlon ingest $\sim 3,643 \mathrm{kcal}$ and expend $\sim 11,009 \mathrm{kcal}$ leading to an energy deficit of $\sim 7,365 \mathrm{kcal} .{ }^{70}$ Apart from fluid and electrolyte metabolism, carbohydrate depletion during an Ironman triathlon might also impair performance. Overall Ironman race time was inversely related to carbohydrate intake during the marathon in male Ironman triathletes but not for female athletes. ${ }^{71}$ In both female and male Ironman triathletes, total carbohydrate intake rates were negatively correlated to overall race time. ${ }^{69}$ An increased carbohydrate intake during the marathon might also be a useful strategy for improving Ironman performance in male triathletes. ${ }^{71}$ 


\section{Neuromuscular fatigue}

An Ironman triathlon is associated with changes in body composition (ie, decrease in fat and muscle mass) as well as decreases in neuromuscular function. Peak power, peak velocity, jump height, and rate of force development decreased during an Ironman triathlon. ${ }^{81}$ Total and positive impulses during a countermovement jump were reduced after the triathlon, while both negative impulses were not different before and after the Ironman. ${ }^{81}$ Absolute peak force remained constant during countermovement jump and squat jump. ${ }^{81}$ Maximal voluntary ground reaction force and peak stiffness during multiple one-legged hopping were decreased after the Ironman. ${ }^{81}$ The neuromuscular deficit after the Ironman race was due to impairments in force transmission, resulting in a lower average positive force during countermovement jump because of a slower rate of force development. ${ }^{81}$ An idea to prevent muscular problems could be the use of compression socks. However, Del Coso et al demonstrated for Half-Ironman triathletes that wearing compression stockings showed no advantage for maintaining muscle function or reducing blood markers of muscle damage. ${ }^{82}$

\section{Limitations and perspectives}

Data from the selected studies were mainly obtained from subelite or recreational athletes. Data from elite athletes

Table 4 Propose of a new profile of winners

\begin{tabular}{|c|c|}
\hline Female & Male \\
\hline Origin from United States $>$ Great & Origin from United States $>$ \\
\hline Britain $>$ Switzerland & Germany $>$ Australia \\
\hline Age $32-33$ years & Age $32-33$ years \\
\hline Fast personal best time in Olympic & Fast personal best time in \\
\hline \multirow[t]{2}{*}{ distance triathlon ( $\leq 2: 10 \mathrm{~h}: \mathrm{min})$} & Olympic distance triathlon \\
\hline & $(\leq 2: 32 \mathrm{~h}: \min )$ \\
\hline Fast personal best time in marathon & Fast personal best time in \\
\hline running ( $\leq 3: 13 \mathrm{~h}: \mathrm{min})$ & marathon running ( $\leq 3: 50 \mathrm{~h}: \mathrm{min})$ \\
\hline \multicolumn{2}{|l|}{ Low percent body fat $(\leq 13 \%-15 \%$} \\
\hline \multicolumn{2}{|l|}{ body fat) } \\
\hline \multicolumn{2}{|l|}{ Low sum of skin-fold thicknesses } \\
\hline \multicolumn{2}{|l|}{ (eight sites) $(\leq 70 \mathrm{~mm})$} \\
\hline \multicolumn{2}{|l|}{ Low circumference of upper arm } \\
\hline \multicolumn{2}{|l|}{$(\leq 30 \mathrm{~cm})$} \\
\hline \multicolumn{2}{|l|}{ Low circumference of thigh $(\leq 54 \mathrm{~cm})$} \\
\hline \multicolumn{2}{|l|}{ Weekly training hours ( $\leq 14$ hours) } \\
\hline & Weekly swimming kilometers \\
\hline & ( $\leq 7$ km/week) \\
\hline Weekly cycling kilometers & Cycling speed during training \\
\hline ( $\leq 190$ km/week) & $(\leq 28 \mathrm{~km} / \mathrm{h})$ \\
\hline Running speed during training & Running speed during training \\
\hline$(\leq 10.5 \mathrm{~km} / \mathrm{h})$ & $(\leq \mathrm{I} / \mathrm{km} / \mathrm{h})$ \\
\hline High maximum oxygen uptake & High maximum oxygen uptake \\
\hline$\left(\geq 66.7 \mathrm{~mL} \mathrm{~kg}^{-1} \mathrm{~min}^{-1}\right)$ & $\left(\geq 61.6 \mathrm{~mL} \mathrm{~kg}{ }^{-1} \mathrm{~min}^{-1}\right)$ \\
\hline
\end{tabular}

Abbreviations: h, hours; min, minutes. competing at world class level are missing. Future studies should investigate anthropometric and physiological variables from elite Ironman triathletes. A striking finding is that mainly athletes from the "first world" such as United States of America, Germany, Japan, Australia, Canada, Switzerland, France, Great Britain, New Zealand, and Austria compete in qualifiers and in "Ironman Hawaii". 3,44 Future studies need to investigate socioeconomic aspects in Ironman triathlon. Future studies also need to correlate physiological characteristics such as $\mathrm{VO}_{2}$ max with Ironman race time to investigate their predictive value for race performance. For "Ironman Hawaii", the pacing during the split disciplines, especially in cycling and running, might be investigated to understand how these athletes pace during their races. When the personal best marathon time is a strong predictor variable for Ironman race time, future studies might investigate the "history" of Ironman triathletes whether they originate from swimming, cycling, or running.

\section{Conclusion}

The most important predictive variables for a fast Ironman race time were age of 30-35 years (women and men), a fast personal best time in Olympic distance triathlon (women and men), a fast personal best time in marathon (women and men), high volume and high intensity in training where a higher volume was more predictive than a higher intensity (women and men), low body fat, low skin-fold thicknesses and low circumference of upper arm (only men), and origin from the United States of America (women and men). Table 4 presents the most important predictors for potential future Ironman winners. Future studies need to correlate physiological characteristics such as $\mathrm{VO}_{2}$ max with Ironman race performance to investigate whether these characteristics are predictive for race performance.

\section{Disclosure}

The authors report no conflicts of interest in this work.

\section{References}

1. Ironman World Championship. Available from: http://www. ironman.com/triathlon/events/americas/ironman/world-championship. aspx\#axzz3J9L4ftoT. Accessed March 22, 2015.

2. The 12 Hardest Races in the World. Available from: http://www.fitbie. com/slideshow/12-hardest-races-world. Accessed March 22, 2015.

3. Stiefel M, Knechtle B, Rüst CA, Rosemann T. Analysis of performances at the 'Ironman Hawaii triathlon' and its qualifier events with respect to nationality. J Sci Cycling. 2013;2:27-34.

4. Stiefel M, Rüst CA, Rosemann T, Knechtle B. A comparison of participation and performance trends in age group finishers competing in and qualifying for 'Ironman Hawaii'. Int J Gen Med. 2013;6:67-77. 
5. PubMed.gov [database on the Internet]. Bethesda, MD: National Center for Biotechnology Information, US Library of Medicine. Available from: http://www.ncbi.nlm.nih.gov/pubmed. Accessed March 22, 2015.

6. Gilinsky N, Hawkins KR, Tokar TN, Cooper JA. Predictive variables for half-Ironman triathlon performance. J Sci Med Sport. 2014;17: 300-305.

7. Lepers R, Sultana F, Bernard T, Hausswirth C, Brisswalter J. Agerelated changes in triathlon performances. Int J Sports Med. 2010;31: 251-256.

8. Lepers R, Rüst CA, Stapley P, Knechtle B. Relative improvements in endurance performance with age: Evidence from 25 years of Hawaii Ironman racing. Age (Dordr). 2013;35:953-962.

9. Lepers R, Knechtle B, Stapley P. Trends in triathlon performance: effects of sex and age. Sports Med. 2013;43:851-863.

10. Knechtle R, Rüst CA, Rosemann T, Knechtle B. The best triathletes are older in longer race distances - a comparison between Olympic, HalfIronman and Ironman distance triathlon. SpringerPlus. 2014;3:538.

11. Knechtle B, Rüst CA, Rosemann T, Lepers R. Age- and gender differences in half-Ironman triathlon performances - the Ironman 70.3 Switzerland from 2007 to 2010. Open Access J Sports Med. 2012;3: 59-66.

12. Stiefel M, Knechtle B, Rüst CA, Rosemann T, Lepers R. The age of peak performance in Ironman triathlon - a cross-sectional and longitudinal data analysis. Extrem Physiol Med. 2013;2:27.

13. Rüst CA, Knechtle B, Knechtle P, Rosemann T, Lepers R. Age of peak performance in elite male and female Ironman triathletes competing in Ironman Switzerland, a qualifier for the Ironman world championship, 'Ironman Hawaii' from 1995 to 2011. Open Access J Sports Med. 2012;3:175-182.

14. Gallmann D, Knechtle B, Rüst CA, Rosemann T, Lepers R. Elite triathletes in 'Ironman Hawaii' get older but faster. Age (Dordr). 2014;36:407-416.

15. Stiefel M, Knechtle B, Lepers R. Master triathletes have not reached limits in their Ironman triathlon performance. Scand J Med Sci Sports. 2014;24:89-97.

16. Gulbin JP, Gaffney PT. Ultraendurance triathlon participation: typical race preparation of lower level triathletes. J Sports Med Phys Fitness. 1999;39:12-15.

17. Knechtle B, Wirth A, Rosemann T. Predictors of race time in male Ironman triathletes: physical characteristics, training or pre race experience? Percept Mot Skills. 2010;111:437-446.

18. Knechtle B, Wirth A, Baumann B, Knechtle P, Rosemann T. Personal best time, percent body fat and training volume are differently associated with race time in male and female Ironman triathletes. Res $Q$ Exerc Sport. 2010;81:62-68.

19. Rüst CA, Knechtle B, Knechtle P, Rosemann T, Lepers R. Personal best times in an Olympic distance triathlon and a marathon predict Ironman race time in recreational male triathletes. Open Access J Sports Med. 2011;2:121-129.

20. Rüst CA, Knechtle B, Wirth A, et al. Personal best times in an Olympic distance triathlon and a marathon predict an Ironman race time for recreational female triathletes. Chin J Physiol. 2012;55:156-162.

21. Personal website of Nicola Spirig. Available from: http://www. nicolaspirig.ch/Erfolge.6.0.html. Accessed April 22, 2015.

22. Lepers R. Analysis of Hawaii Ironman performances in elite triathletes from 1981 to 2007. Med Sci Sports Exerc. 2008;40:1828-1834.

23. Lepers R, Maffiuletti NA. Age and gender interactions in ultraendurance performance: insight from the triathlon. Med Sci Sports Exerc. 2011;43:134-139.

24. Stevenson JL, Song H, Cooper JA. Age and sex differences pertaining to modes of locomotion in triathlon. Med Sci Sports Exerc. 2013;45: 976-984.

25. Neal CM, Hunter AM, Galloway SD. A 6-month analysis of training-intensity distribution and physiological adaptation in Ironman triathletes. J Sports Sci. 2011;29:1515-1523.
26. O’Toole ML. Training for ultraendurance triathlons. Med Sci Sports Exerc. 1989;21:S209-S213.

27. Knechtle B, Wirth A, Knechtle P, Baumann B, Rosemann T, Senn O. Differential correlations between anthropometry, training volume and performance in male and female Ironman triathletes. J Strength Cond Res. 2010;24:2785-2793.

28. Rüst CA, Knechtle B, Knechtle P, Rosemann T. A comparison of anthropometric and training characteristics between recreational female marathoners and female Ironman triathletes. Chin J Physiol. 2013;56:1-10.

29. Gianoli D, Knechtle B, Knechtle P, Barandun U, Rüst CA, Rosemann T. Comparison between recreational male Ironman triathletes and marathon runners. Percept Mot Skills. 2012;115:283-299.

30. Knechtle B, Knechtle P, Rüst CA, Rosemann T. A comparison of anthropometric and training characteristics among Ironman and Triple Iron triathletes. J Sports Sci. 2011;29:1373-1380.

31. Rüst CA, Knechtle B, Knechtle P, Wirth A, Rosemann T. A comparison of anthropometric and training characteristics among recreational male Ironman triathletes and ultra-endurance cyclists. Chin J Physiol. 2012;55:114-124.

32. Muñoz I, Cejuela R, Seiler S, Larumbe E, Esteve-Lanao J. Trainingintensity distribution during an ironman season: relationship with competition performance. Int J Sports Physiol Perform. 2014;9:332-339.

33. Kandel M, Baeyens JP, Clarys P. Somatotype, training and performance in Ironman athletes. Eur J Sport Sci. 2014;14:301-308.

34. Knechtle B, Wirth A, Rüst CA, Rosemann T. Relationship between anthropometry and split performance in recreational male Ironman triathletes. Asian J Sports Med. 2011;2:23-30.

35. Silva DA, Benedetti TR, Ferrari EP, et al. Anthropometric profiles of elite older triathletes in the Ironman Brazil compared with those of young Portuguese triathletes and older Brazilians. J Sports Sci. 2012;30:479-484.

36. Bernheim A, Attenhofer Jost $\mathrm{CH}$, et al. The right ventricle best predicts the race performance in amateur Ironman athletes. Med Sci Sports Exerc. 2013;45:1593-1599.

37. Knechtle B, Knechtle P, Rosemann T. Upper body skin-fold thickness is related to race performance in male Ironman triathletes. Int $J$ Sports Med. 2011;32:20-27.

38. Del Coso J, González C, Abian-Vicen J, et al. Relationship between physiological parameters and performance during a half-ironman triathlon in the heat. J Sports Sci. 2014;32:1680-1687.

39. Knechtle B, Wirth A, Baumann B, et al. An ironman triathlon does not lead to a change in body mass in female triathletes. Res Sports Med. 2010;18:115-126.

40. Knechtle B, Baumann B, Wirth A, Knechtle P, Rosemann T. Male Ironman triathletes lose skeletal muscle mass. Asia Pac J Clin Nutr. 2010;19:91-97.

41. Mueller SM, Anliker E, Knechtle P, Knechtle B, Toigo M. Changes in body composition in triathletes during an Ironman race. Eur J Appl Physiol. 2013;113:2343-2352.

42. Cuddy JS, Slivka DR, Hailes WS, Dumke CL, Ruby BC. Metabolic profile of the Ironman World Championships: a case study. Int J Sports Physiol Perform. 2010;5:570-576.

43. The Ironman Story, available from: http://www.ironman.com/triathlon/ history.aspx\#axzz3J9L4ftoT. Accessed March 22, 2015.

44. Dähler P, Rüst CA, Rosemann T, Lepers R, Knechtle B. Nation related participation and performance trends in 'Ironman Hawaii' from 1985 to 2012. BMC Sports Sci Med Rehabil. 2014;6:16.

45. Sleivert GG, Rowlands DS. Physical and physiological factors associated with success in the triathlon. Sports Med. 1996;22:8-18.

46. O’Toole ML, Hiller DB, Crosby LO, Douglas PS. The ultraendurance triathlete: a physiological profile. Med Sci Sports Exerc. 1987;19: $45-50$.

47. Leischik R, Spelsberg N. Endurance sport and "cardiac injury": a prospective study of recreational ironman athletes. Int J Environ Res Public Health. 2014;11:9082-9100. 
48. Hue O. Prediction of drafted-triathlon race time from submaximal laboratory testing in elite triathletes. Can J Appl Physiol. 2003;28:547-560.

49. Laursen PB, Rhodes EC. Factors affecting performance in an ultraendurance triathlon. Sports Med. 2001;31:195-209.

50. Laursen PB, Rhodes EC, Langill RH, McKenzie DC, Taunton JE. Relationship of exercise test variables to cycling performance in an Ironman triathlon. Eur J Appl Physiol. 2002;87:433-440.

51. O'Toole ML, Douglas PS, Hiller WD. Lactate, oxygen uptake, and cycling performance in triathletes. Int J Sports Med. 1989;10:413-418.

52. Schabort EJ, Killian SC, St Clair Gibson A, Hawley JA, Noakes TD Prediction of triathlon race time from laboratory testing in national triathletes. Med Sci Sports Exerc. 2000;32:844-849.

53. Whyte G, Lumley S, George K, et al. Physiological profile and predictors of cycling performance in ultra-endurance triathletes. J Sports Med Phys Fitness. 2000;40:103-109.

54. Puggina EF, Machado DR, Tourinho Filho H, Barbanti VJ. Halfironman induces changes in the kidney function of triathletes. An Acad Bras Cienc. 2014;86:429-436.

55. Broadbent S. Seasonal changes in haematology, lymphocyte transferrin receptors and intracellular iron in Ironman triathletes and untrained men. Eur J Appl Physiol. 2011;111:93-100.

56. Holly RG, Barnard RJ, Rosenthal M, Applegate E, Pritikin N. Triathlete characterization and response to prolonged strenuous competition. Med Sci Sports Exerc. 1986;18:123-127.

57. Wu SS, Peiffer JJ, Brisswalter J, Nosaka K, Abbiss CR. Factors influencing pacing in triathlon. Open Access J Sports Med. 2014;5:223-234.

58. Johnson EC, Pryor JL, Casa DJ, et al. Bike and run pacing on downhill segments predict Ironman triathlon relative success. J Sci Med Sport. 2015;18:82-87.

59. Sharwood K, Collins M, Goedecke J, Wilson G, Noakes T. Weight changes, sodium levels, and performance in the South African Ironman Triathlon. Clin J Sport Med. 2002;12:391-399.

60. Pahnke MD, Trinity JD, Zachwieja JJ, Stofan JR, Hiller WD, Coyle EF. Serum sodium concentration changes are related to fluid balance and sweat sodium loss. Med Sci Sports Exerc. 2010;42: 1669-1674.

61. Rüst CA, Knechtle B, Knechtle P, Rosemann T. Higher prevalence of exercise-associated hyponatremia in triple iron ultra-triathletes than reported for ironman triathletes. Chin J Physiol. 2012;55:147-155.

62. Speedy DB, Noakes TD, Rogers IR, et al. Hyponatremia in ultradistance triathletes. Med Sci Sports Exerc. 1999;31:809-815.

63. Speedy DB, Rogers IR, Noakes TD, et al. Exercise-induced hyponatremia in ultradistance triathletes is caused by inappropriate fluid retention. Clin J Sport Med. 2000;10:272-278.

64. Speedy DB, Rogers IR, Noakes TD, et al. Diagnosis and prevention of hyponatremia at an ultradistance triathlon. Clin J Sport Med. 2000;10:52-58.

65. Speedy DB, Noakes TD, Rogers IR, et al. A prospective study of exercise-associated hyponatremia in two ultradistance triathletes. Clin J Sport Med. 2000;10:136-141.

66. Speedy DB, Noakes TD, Kimber NE, et al. Fluid balance during and after an ironman triathlon. Clin J Sport Med. 2001;11:44-50.

67. Speedy DB, Noakes TD, Boswell T, Thompson JM, Rehrer N, Boswell DR. Response to a fluid load in athletes with a history of exercise induced hyponatremia. Med Sci Sports Exerc. 2001;33: 1434-1442.

68. Speedy DB, Thompson JM, Rodgers I, Collins M, Sharwood K, Noakes TD. Oral salt supplementation during ultradistance exercise. Clin J Sport Med. 2002;12:279-284.

69. Pfeiffer B, Stellingwerff T, Hodgson AB, et al. Nutritional intake and gastrointestinal problems during competitive endurance events. Med Sci Sports Exerc. 2012;44: 344-351.
70. Barrero A, Erola P, Bescós R. Energy balance of triathletes during an ultra-endurance event. Nutrients. 2014;7:209-222.

71. Kimber NE, Ross JJ, Mason SL, Speedy DB. Energy balance during an ironman triathlon in male and female triathletes. Int J Sport Nutr Exerc Metab. 2002;12:47-62.

72. Robins A. Nutritional recommendations for competing in the Ironman triathlon. Curr Sports Med Rep. 2007;6:241-248.

73. Hiller WD. Dehydration and hyponatremia during triathlons. Med Sci Sports Exerc. 1989;21:S219-S221.

74. Noakes TD, Sharwood K, Collins M, Perkins DR. The dipsomania of great distance: water intoxication in an Ironman triathlete. Br J Sports Med. 2004;38:E16.

75. Richter S, Betz C, Geiger H. Severe hyponatremia with pulmonary and cerebral edema in an Ironman triathlete. Dtsch Med Wochenschr. 2007;132:1829-1832.

76. Severac M, Orban JC, Leplatois T, Ichai C. A near-fatal case of exerciseassociated hyponatremia. Am J Emerg Med. 2014;32:813. e1-813. e2.

77. Stefanko G, Lancashire B, Coombes JS, Fassett RG. Pulmonary oedema and hyponatraemia after an ironman triathlon. BMJ Case Rep. Epub August 17, 2009.

78. Meyer M, Knechtle B, Bürge J, et al. Ad libitum fluid intake leads to no leg swelling in male Ironman triathletes - an observational field study. J Int Soc Sports Nutr. 2012;9:40.

79. Laursen PB, Watson G, Abbiss CR, Wall BA, Nosaka K. Hyperthermic fatigue precedes a rapid reduction in serum sodium in an ironman triathlete: a case report. Int J Sports Physiol Perform. 2009;4:533-537.

80. Del Coso J, González-Millán C, Salinero JJ, et al. Effects of oral salt supplementation on physical performance during a half-ironman: a randomized controlled trial. Scand JMed Sci Sports. Epub February 14, 2015.

81. Mueller SM, Knechtle P, Knechtle B, Toigo M. An Ironman triathlon reduces neuromuscular performance due to impaired force transmission and reduced leg stiffness. Eur J Appl Physiol. 2015;115:795-802.

82. Del Coso J, Areces F, Salinero JJ, et al. Compression stockings do not improve muscular performance during a half-ironman triathlon race. Eur J Appl Physiol. 2014;114:587-595.

83. Personal website of Sebastian Kienle. Available from: http://www. sebastiankienle.de. Accessed April 22, 2015.

84. Personal website of Frederik Van Lierde. Available from: http://www. frederikvanlierde.com. Accessed April 22, 2015.

85. Personal website of Pete Jacobs. Available from: http://www.petejacobs. com. Accessed April 22, 2015.

86. Personal website of Craig Alexander. Available from: http://www. craigalexander.net. Accessed April 22, 2015.

87. Personal website of Chris McCormack. Available from: http://www. chrismccormack.com. Accessed April 22, 2015.

88. Personal website of Norman Stadler. Available from: http:/www. normann-stadler.de. Accessed April 22, 2015.

89. Personal website of Faris Al-Sultan. Available from: http://www.farisal-sultan.de. Accessed April 22, 2015.

90. Personal website of Mirinda Carfrae. Available from: http:// mirindacarfrae.com. Accessed April 22, 2015.

91. Personal website of Leanda Cave. Available from: http://leandacave. com. Accessed April 22, 2015.

92. Personal website of Chrissie Wellington. Available from: http://www. chrissiewellington.org. Accessed April 22, 2015.

93. International Triathlon Union. [webpage on the Internet]. Race results for Michellie Jones. Available from: http://www.triathlon.org/athletes/ results/5499/michellie_jones. Accessed April 22, 2015.

94. SportsStats World. [webpage on the Internet]. Race results for Natascha Badmann. http://www.9stunden.com/?id=2. Accessed April 22, 2015. 


\section{Publish your work in this journal}

Open Access Journal of Sports Medicine is an international, Visit http://www.dovepress.com/testimonials.php to read real quotes peer-reviewed, open access journal publishing original research, from published authors.

reports, reviews and commentaries on all areas of sports

medicine. The manuscript management system is completely

online and includes a very quick and fair peer-review system.

Submit your manuscript here: http://www.dovepress.com/open-access-journal-of-sports-medicine-journal 